\title{
Künstliche Intelligenz: Was Menschen und Maschinen lernen müssen
}

\author{
Künstliche Intelligenz (KI) polarisiert - genauso \\ wie die Erfindung der Eisenbahn vor fast \\ 200 Jahren. Doch zwischen Euphorie und totaler \\ Ablehnung ist viel Raum für den kreativen \\ Umgang mit neuen Technologien. Wirtschaft, \\ Wissenschaft und Politik sollten ihn rasch \\ nutzen.
}

Die KI kommt. Und schon verfällt die Menschheit in die bekannten Reflexe: Die einen feiern ein neues Zeitalter, in der „die KI“ alle Probleme der Menschheit löst. Die anderen warnen vor den Risiken - echten und eingebildeten. Dabei schaden beide Gruppen der Sache, weil sie eine vernünftige, an Machbarkeit und Nutzen orientierte Auseinandersetzung mit der Technologie behindern. Das Problem dabei: Die Entwicklung läuft bereits. Auch ohne uns als Deutsche und Europäer. Aber wir können es uns nicht leisten, sie einfach laufen $\mathrm{zu}$ lassen. Schauen wir also genauer hin.

\section{Künstliche Intelligenz ist nicht gleich künst- liche Intelligenz}

Zunächst müssen wir unterscheiden - zwischen „starker“ und „schwacher“ KI. Starke KI kennen wir aus Science-Fiction-Märchen. Es ist „die“ KI, die ähnlich wie ein Mensch eine Vielzahl von Anwendungsbereichen beherrscht. Allerdings hat sie einen großen Nachteil: Es gibt sie nicht. Was es hingegen gibt, sind Anwendungen von KI-Algorithmen auf einzelne Bereiche, wie etwa Text- oder Bilderkennung. Tatsächlich arbeiten Forscherinnen und Forscher an Universitäten und in Unternehmen daran seit vielen Jahren. Zurzeit jedoch erleben wir weltweit einen großen Entwicklungsschub im Zuge der Digitalisierung. Das liegt vor allem daran, dass Rechenkapazität, Vernetzungsgrad und Speicherfähigkeit der IT-Systeme enorme Fortschritte gemacht haben. So wurde es möglich, dass viele Konzepte der schwachen KI, die in der Theorie schon länger bestehen, heute praktisch umsetzbar sind, etwa in den Bereichen Datenanalysen, Bilderkennung, Texterkennung und Übersetzung. Wie groß das Potenzial der bereits vorhandenen Technologien im Bereich maschinelles Lernen ist und wie rasch es sich erschließen lässt, wenn wir

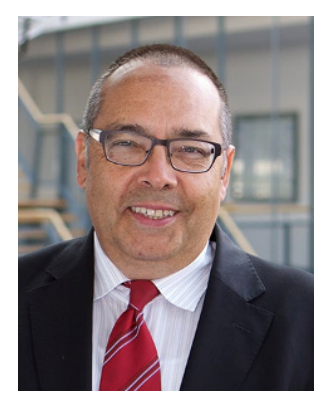

Prof. Dr. Helmut Krcmar ( $\triangle)$ ${ }^{*}{ }^{1954)}$ ist Inhaber des Lehrstuhls für Wirtschaftsinformatik an der Fakultät für Informatik, Vice Dean TUM School of Management und Gründungsdekan des TUM Campus Heilbronn der Technischen Universität München (TUM) sowie Vorstandsmitglied und Vorsitzender des Forschungsausschusses im MÜNCHNER KREIS e. V. Er forscht auf dem Gebiet der digitalen Transformation, des Informations- und Wissensmanagements, der plattformbasierten Ökosysteme, des Managements IT-basierter Dienstleistungen und des E-Governments und der Computerunterstützung für die Kooperation.

helmut.krcmar@tum.de

TU München, München, Deutschland

die vorhandene Technologie ohne ideologische Scheuklappen erforschen und weiterentwickeln, das lässt sich am besten anhand konkreter Anwendungsbeispiele veranschaulichen, wie sie der MÜNCHNER KREIS e. V. auf seinen Veranstaltungen zum Thema immer wieder präsentiert - zuletzt im Rahmen der Veranstaltung „Künstliche Intelligenz und die Automation des Entscheidens“. Mehr als 200 Teilnehmer aus Wirtschaft

Wirtschaftsinformatik \& Management 2020 • 12 (2): 114-115 https://doi.org/10.1365/s35764-020-00252-4

Online publiziert: 12. März 2020

(c) Der/die Autor(en) 2020 
und Wissenschaft erfuhren dort unter anderem, wie wichtig aktuelle Produktionsdaten sind, damit KI die Entwicklung der Stromproduktion in Windkraftanlagen mit einer Präzision von $91 \%$ vorhersagen kann. Tatsächlich lag die Vorhersagegenauigkeit ohne diese Daten nur bei $68 \%$. Das zeigt: die Intelligenz der Systeme hängt wesentlich von den Daten ab, mit denen wir, die Menschen, sie füttern.

\section{Die Verantwortung bleibt bei den Menschen}

Wir Menschen bleiben in der Verantwortung für die Entscheidungen, die solche schwachen KI-Anwendungen treffen. Folglich müssen wir uns darum kümmern, dass wir über die Mittel verfügen, um diese Verantwortung wahrzunehmen. Dazu gehören neben der Fähigkeit, immer mehr Informationen zu erfassen und zu verarbeiten vor allem Datenschutz und Datensicherheit. Allerdings müssen wir in all diesen Bereichen sehr schnell sehr viel besser werden, wenn wir die Entwicklung von KI-Technologien weiterhin mitgestalten wollen.

Warum das so wichtig ist, zeigt sich im Zusammenhang einer KI-Anwendung bei Siemens. Um 20 \% reduzieren KI-basierte Datenanalysen dort die Stickstoffemissionen von Gasturbinen, indem sie helfen, deren Betrieb zu optimieren. Das Projekt macht aber auch deutlich, dass KI letztlich immer im Umfeld ihrer Anwendung gesehen werden muss. Im konkreten Fall geht es darum, dass die "Intelligenz" im Internet der Dinge (IoT) nicht mehr in der Cloud arbeitet, sondern am Edge, in den Turbinen. Denn genau hier müssen die Entscheidungen über die Veränderung von Parametern so schnell fallen, dass keine Zeit mehr bleibt für den Datentransfer in die Cloud und wieder zurück. Das gilt in vielen Industrieprozessen genauso wie beispielsweise für autonome Fahr- oder Flugzeuge. Wenn Deutschland und Europa in den Schlüsselbranchen ihrer Wirtschaft wettbewerbsfähig bleiben und nicht nur Teile zuliefern wollen, müssen wir in der Lage sein, KI-basierte Systeme auf dem jeweils neuesten Stand der Technik zu entwickeln und herzustellen. Darüber hinaus werden maschinelles Lernen und andere KI-Konzepte künftig auch Betrieb, Wartung und Instandsetzung prägen. Nur so sind beispielsweise Plattformen realisierbar, die Mobilität als Service von Haus zu Haus bieten und dabei unterschiedliche Verkehrsmittel so kombinieren, dass die Reise so schnell, komfortabel und umweltschonend wie möglich abläuft. Das erfordert KI-basierte Steuerungssysteme sowohl in der Cloud als auch am Edge.
Zugegeben: Bis es so weit ist, bleibt eine ganze Menge zu tun. Eine große Aufgabe ist der Bau einer leistungsfähigen Infrastruktur für die Datenübertragung zwischen Anwendungen in der Cloud und den Endgeräten sowie zwischen den Endgeräten untereinander. So braucht KI in der Landwirtschaft beispielsweise $5 \mathrm{G}$ eben auch abseits von Ballungszentren und Autobahnen. Außerdem gibt es immer noch Berechnungen, bei denen die heutige Computertechnik an ihre Grenzen stößt. Offen ist derzeit, ob und wie Quanten-Computing hier helfen kann. Vor allem aber müssen KI-Anwendungen lernen, wann welche Instanz wie entscheiden darf und wann menschliches Eingreifen erforderlich ist. Und wir selbst müssen lernen, dass KI selbstverständlicher Bestandteil des Alltags wird. Einige interessante Lerneinheiten dazu plant der MÜNCHNER KREIS übrigens dieses Jahr in der Fachkonferenz „KI ${ }^{4}$ : Mensch, Führung, Industrie und Politik“ zu präsentieren.

Funding. Open Access funding provided by Projekt DEAL.

Open Access. Dieser Artikel wird unter der Creative Commons Namensnennung 4.0 International Lizenz veröffentlicht, welche die Nutzung, Vervielfältigung, Bearbeitung, Verbreitung und Wiedergabe in jeglichem Medium und Format erlaubt, sofern Sie den/die ursprünglichen Autor(en) und die Quelle ordnungsgemäß nennen, einen Link zur Creative Commons Lizenz beifügen und angeben, ob Änderungen vorgenommen wurden.

Die in diesem Artikel enthaltenen Bilder und sonstiges Drittmaterial unterliegen ebenfalls der genannten Creative Commons Lizenz, sofern sich aus der Abbildungslegende nichts anderes ergibt. Sofern das betreffende Material nicht unter der genannten Creative Commons Lizenz steht und die betreffende Handlung nicht nach gesetzlichen Vorschriften erlaubt ist, ist für die oben aufgeführten Weiterverwendungen des Materials die Einwilligung des jeweiligen Rechteinhabers einzuholen.

Weitere Details zur Lizenz entnehmen Sie bitte der Lizenzinformation auf http://creativecommons.org/licenses/by/4.0/ deed.de.

Springer Professional $\mid \begin{aligned} & \text { Mehr zum Thema finden Sie online } \\ & \text { www.springerprofessional.de/wum }\end{aligned}$ 